doi: $10.2306 /$ scienceasia1513-1874.2013.39.615

\title{
Proteins that interact with rice pumilio 1
}

\author{
Yuyun Sugiharti ${ }^{\mathrm{a}}$, Aqil Azizi ${ }^{\mathrm{b}}$, Mukhamad Su'udi ${ }^{\mathrm{a}, \mathrm{c}, *}$ \\ a Department of Integrative Plant Science, Chung-Ang University, Anseong 456-756, Korea \\ b Department of Marine Biotechnology, University of Science and Technology, Daejeon 305-333, Korea \\ c Department of Agricultural Biotechnology, National Academy of Agricultural Science, RDA, \\ Suwon 441-707, Korea \\ *Corresponding author, e-mail: msuudi.rda@gmail.com
}

Received 5 Nov 2012

Accepted 4 Aug 2013

\begin{abstract}
The N-terminal region of rice pumilio 1 fused with the binding domain (BD-OsPUM1) was used as a bait construct in yeast two-hybrid screening with a rice cDNA library as prey. Several interacting proteins were screened in a stringent media and tested with beta-galactose filter assay. The nucleotide sequences encoding interacting proteins were determined and annotated according to the rice genome database. These proteins are sigma factor F inhibitor, RPL18C, RUBQ2, RPL24A, RCY1, small nuclear ribonucleoprotein G, transferase hexapeptide repeat-containing protein, dormancyassociated protein, and putative expressed proteins with the accessions NP_001067038 and EEE55952. This study suggested that OsPUM1 protein is associated with several biological processes involved in morphology determination, protein folding and plant immunity.
\end{abstract}

KEYWORDS: interacting proteins, OsPUM1, yeast two-hybrid assay

\section{INTRODUCTION}

Pumilio (PUM) proteins are a class of RNA-binding proteins harbouring specific repeated motifs called the Puf domains. These proteins have been reported to play essential roles in several biological processes. For example, Puf3p is involved in mitochondrial biogenesis in yeast ${ }^{1}$. In budding yeast, Puf4p and Puf5p control post translational processes by binding and removing the poly(A) tail of the target mRNAs ${ }^{2}$. Further studies in higher organisms, such as Drosophila, Caenorhabditis elegans, and humans, indicated that PUM proteins are indispensable for maintaining the germ-line stem cell identity ${ }^{3-5}$. In contrast to abundant information about PUM proteins obtained in yeast and animals, functional studies in plants are lacking.

Recently, by taking advantages of the completed genome and availability of T-DNA mutant lines in Arabidopsis, researchers have begun to explore the possible functions of PUM proteins in plants. Arabidopsis pumilio 1, 2, and 3 (APUM1, 2, 3) have been reported to play roles in maintaining plant stem cell identity and differentiation ${ }^{6}$. Specific expression of APUM10 in the apical cell layers of embryo occur at the heart-shape stage ${ }^{7}$. Using a similar approach, APUM16 and APUM17 showed specific expression in the sperm cells, suggesting their role in cell divi$\operatorname{sion}^{8}$. Furthermore, APUM23 is critical for normal growth patterning and development in Arabidopsis ${ }^{9}$. However, in monocot plants such as rice, the functions of PUM proteins are not well understood.

The yeast two-hybrid system $(\mathrm{Y} 2 \mathrm{H})$ is one of the most powerful and versatile methods to study proteinprotein interaction as well as characterizing protein function. This system identifies interacting proteins through the functional restoration of the yeast GAL4 transcriptional activator in vivo ${ }^{10}$. In the study of PUM proteins, the $\mathrm{Y} 2 \mathrm{H}$ system has been utilized for screening the interacting partners of PUM2 in human ${ }^{5}$.

In this study, the $\mathrm{Y} 2 \mathrm{H}$ system was employed to identify proteins that interact specifically with rice Pumilio 1 (OsPUM1). The N-terminal region was used as a bait for screening, and a rice cDNA library was utilized as the prey. The primary information obtained from this study will provide new insights for further studies.

\section{MATERIALS AND METHODS}

\section{Sequence and phylogenetic tree analysis}

The nucleotide and amino acid sequences of OsPUM1 were retrieved from the Knowledge-based Oryza Molecular Biology Encyclopedia ${ }^{11}$ (cdna01.dna.affrc. go.jp/cDNA/). The accession number of OsPUM1 cDNA was AK070870. Alignment between cDNA and genomic DNA was observed using SPIDEY ${ }^{12}$ 
(www.ncbi.nlm.nih.gov/spidey/). Molecular weight and isoelectric point (pI) was calculated using the prediction site ${ }^{13}$ : web.expasy.org/compute_pi/. In addition, protein domain features were determined using Prosite ${ }^{14}$ (www.expasy.org/prosite/). The phylogenetic tree was constructed using algorithms from CLUSTALW in EMBL-EBI website (www.ebi.ac.uk).

\section{Yeast two-hybrid assay}

For bait vector construction, the $5^{\prime}$ terminal region of OsPUM1 was PCR-amplified using Pfu DNA polymerase with the following primers: $5^{\prime}$-AAT GGC TAC AGA GAG TGC TCG GC- $3^{\prime}$ and $5^{\prime}$-TGT CGA CGG TTG GAA TCC CTT GAC AT- $3^{\prime}$ (the start codon and SalI site is underlined). The pGBT9 vector was sequentially digested with SmaI and SalI. Subsequently, the PCR-amplified product of OsPUMI was digested with SalI and ligated into pGBT9 vector to create pGBT9-OSPUM1. The cDNA library (prey) was made from rice panicle according to the HybriZAP two-hybrid cDNA Gigapack cloning kit manual (Stratagene, La Jolla, CA, USA). Total cDNA of the phagemid form was obtained by the mass in vivo excision method. The yeast (Saccharomyces cerevisiae) strain YRG-2 (genotype: MATa, ura3-52, his3200, ade2-101, lys2-801, trp1-901, leu2-3, 112, gal4542, gal80-538, LYS::UAS $\mathrm{UAL}_{\mathrm{GA}}-\mathrm{TATA}_{\mathrm{GAL} 1}$ - HIS3, URA3::UAS $\mathrm{UAL417mers}(\mathrm{x} 3)_{\mathrm{G})} \mathrm{TATA}_{\mathrm{CYC1}}$-lacZ) was transformed with the binding domain pGBT9-OSPUM1. The yeast cells containing the binding domain were co-transformed with $100 \mu \mathrm{g}$ of the HybriZAP cDNA library plasmid DNA and salmon sperm carrier DNA by the lithium acetate method ${ }^{15}$. Transformants were selected on the synthetic dropout medium lacking tryptophan, leucine, and histidine (SD-TLH). The transformants, which appeared after 3-5 days incubation at $30^{\circ} \mathrm{C}$, were then grown on an SD-TLH plate containing $5 \mathrm{mM}$ and then $25 \mathrm{mM} 3$-aminotriazole (3-AT). Yeast colonies which still survived on media containing $25 \mathrm{mM} \mathrm{3}$-AT were tested for the betagalactosidase activity by the filter assay ${ }^{16}$. The colonies that turned blue in less than $6 \mathrm{~h}$ were collected and used as the template in colony PCR reaction as described previously ${ }^{17}$. The primers for amplification were GAL4 AD-forward and -reverse with following PCR procedure: the denaturation condition was $7 \mathrm{~min}$ at $95^{\circ} \mathrm{C}$, followed by the amplification reaction for $1 \mathrm{~min}$ at $95^{\circ} \mathrm{C}, 1 \mathrm{~min}$ at $55^{\circ} \mathrm{C}$, and $3 \mathrm{~min}$ at $72^{\circ} \mathrm{C}$ for 35 cycles, prior to a final extension for $7 \mathrm{~min}$ at $72^{\circ} \mathrm{C}$ and storage at $4{ }^{\circ} \mathrm{C}$. The PCR product was then purified with a QIAEX II kit (Qiagen, Valencia, CA, USA) and confirmed by DNA sequencing. Nucleotide sequences obtained from

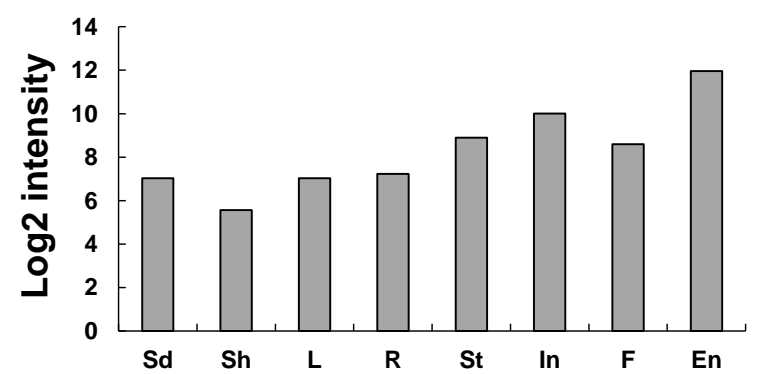

Fig. 1 Expression profile of the OSPUM1 collected from public microarray database (www.ricearray.org). The expression was obtained from Affymatrix meta-analysis using Os.13605.1.S1_at as a main probe set. Sd, seedling; Sh, shoot; L, leaf; R, root; St, stem; In, internode; F, flower; En, endosperm.

DNA sequencing were used for gene annotation by BLAST against NCBI (www.ncbi.nlm.nih.gov) and rice genome database (rice.plantbiology.msu.edu).

\section{RESULTS}

\section{Expression profile, molecular structure and phylogenetic tree of $O S P U M 1$}

OsPUM1 expression was observed from the rice oligonucleotide array database (www.ricearray.org). Through Affymatrix microarray meta-analysis using a main probe specific to OSPUMI (Os.13 605.1.S1_at), the expression was determined in several organs that represents a whole life cycle of rice. OsPUMI was expressed in all organs throughout the life cycle of rice (Fig. 1). The highest transcript level was found in the endosperm, while moderate levels were observed in the internode, stem, and flower. Low expression was detected in the root, seedling, leaf, and shoot (Fig. 1).

The structure of the OSPUMl gene was determined by the sequence information from KOME fulllength cDNA clone (Accession AK070870) and rice genome database (rice.plantbiology.msu.edu). OsPUM1 was located at chromosome number 9 within the rice genome. This gene comprises nine exons and eight introns. The open reading frame of the OsPUM1 cDNA consists of 2967 nucleotides and encodes a protein consisting of 988 amino acids with a predicted molecular mass $(\mathrm{Mw})$ of $107.77 \mathrm{kDa}$ and isoelectric point (pI) of 6.1 (Fig. 2a, b). Eight repeats of the Pumilio domain were located between amino acids 651 and 945 at the C-terminal region of the OsPUM1 protein (Fig. 2b). In contrast, no conserved motif was observed in the $\mathrm{N}$-terminal region. When the OsPUM1 amino acid sequence was compared with known sequences from other plants, it had ap- 


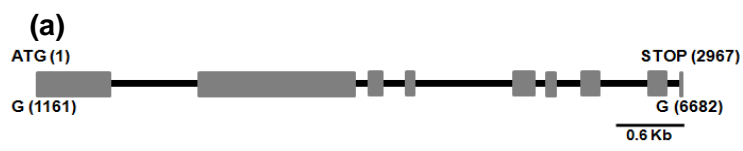

(b)

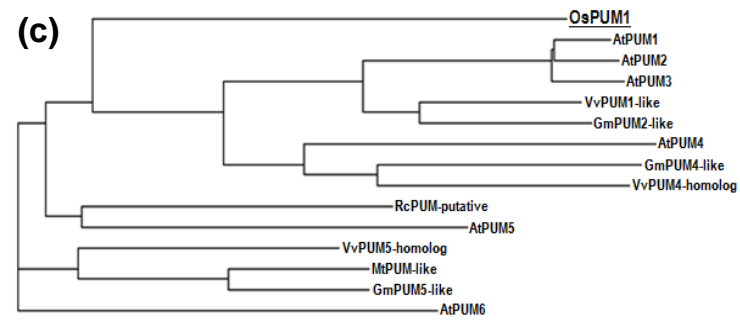

Fig. 2 Structure of the rice OsPUM1 gene and protein and relationship of OsPUM1 to other proteins; (a) molecular structure of the OSPUM1 gene with nine exons (greyfilled boxes) and eight introns (inter-lines); (b) map of the OsPUM1 protein showing pumilio motif (hexagons with $\mathrm{P}$ letters) in C-terminal region and the segments of low-complexity regions (black colours) in N-terminal region; (c) phylogenetic relationships between OsPUM1 (underlined) and other PUM proteins. Phylogenetic tree shows a graphical representation of evolutionary relationships and was constructed with the EBI-CLUSTALW algorithm. Species names, proteins and accession numbers are Arabidopsis thaliana pumilio 1, AtPUM1 (NP_180483); A. thaliana pumilio 2, AtPUM2 (NP_180482); A. thaliana pumilio 3, AtPUM3 (NP_180478); A. thaliana pumilio 4, AtPUM4 (NP_187647); A. thaliana pumilio 5, AtPUM5 (NP_188660); A. thaliana pumilio 6, AtPUM6 (NP_567 733); Glycine max pumilio 2, GmPUM2 (XP_003537980); G. max pumilio 4, GmPUM4 (XP_003535521); G. max pumilio 5, GmPUM5 (XP_003547443); Medicago truncatula pumilio, MtPUM (XP_003594978); Ricinus communis pumilio, RcPUM (XP_002513314); Vitis vinifera pumilio 1, VvPUM1 (XP_002283191); V. vinifera pumilio 4, VvPUM4 (XP_002273503); V. vinifera pumilio 5, VvPUM5 (XP_002 268751).

proximately $28-39 \%$ identity. OsPUM1 shared 39 and $35 \%$ amino acid sequence identity with RcPUM and AtPUM5, respectively, and shared lower identity (28\%) with AtPUM4 and GmPUM4. The evolutionary relationship between these proteins is illustrated in the neighbour joining phylogenetic tree in Fig. 2c.

\section{Screening proteins that could interact with OsPUM1}

The yeast two-hybrid screening was conducted to identify proteins that interact with rice pumilio 1 (Os-
PUM1). The fusion between the GAL4 binding domain and the N-terminal region of OsPUM1 was prepared as a bait construct. Subsequently, the rice cDNA library was introduced into the YRG-2 yeast strain harbouring the bait protein. Selection was performed in SD-Trp-Leu-His (SD-TLH) agar media containing $25 \mathrm{mM}$ 3-aminotriazole (3-AT). The transformants were also tested for $L a c Z$ activation. Out of the 14 colonies grown in the high stringency media, only 11 colonies appeared in blue colour ( $\mathrm{LacZ}$ activation). The colonies putatively harbouring the sequences of interest were then isolated and annotated according to the BLASTn results against NCBI nucleotide database or the rice genome database (rice.plantbiology.msu. $\mathrm{edu} /$ ) and summarized in Table 1. These proteins are sigma factor $\mathrm{F}$ inhibitor (LOC_Os01 g55770), 60S ribosomal protein L18 (RPL18C, LOC_Os03g22 180), 60S ribosomal protein L24 (RPL24A, LOC Os07g12250), polyubiquitin (RUBQ2, LOC_Os02 g06640), ania-6a type cyclin (RCY1, LOC_Os01g27 940), small nuclear ribonucleoprotein G (LOC_Os07 g41790), dormancy-associated protein (LOC_Os11 g44810), transferase hexapeptide repeat-containing protein (LOC_Os01g18070), and two expressed proteins (LOC_Os12g37650 and LOC_Os01 g70670). Among these, the dormancy-associated protein appeared twice during the screening process.

\section{DISCUSSION}

The expression pattern of OsPUM1 was observed from the rice microarray database. From several organs or tissues examined, the expression was relatively high in the endosperm and internodes. This result indicates that OsPUM1 might play a potential role not only during the vegetative stage but also during the period of reproductive development. In addition, the expression of OsPUM1 was detected in all organs or tissues examined, suggesting a broad extent of its function.

Typically, proteins interact with other molecules to regulate their functions. These molecules can be proteins, nucleic acids, lipids, or any other biomolecules that may serve as ligand, substrate, inhibitor, cofactor, co-enzyme or activator. Since the C-terminal region of OsPUM1 is highly conserved among plant species, the N-terminal part was utilized as bait construct in yeast two-hybrid ( $\mathrm{Y} 2 \mathrm{H})$ analysis. By using this strategy, several proteins that interact with OsPUM1 were isolated. One of the proteins that interact with OsPUM1 in rice is a homologue of sigma factor $F(S i g F)$ inhibitor. In bacteria, this protein had been reported to be involved in the regulation of tolerance and susceptibility to rifampin, a drug 
Table 1 List of proteins interact with OsPUM1.

\begin{tabular}{llcc}
\hline Colony number & Gene annotation/putative role & Locus number & Accession number (protein) \\
\hline 1 & Sigma factor F inhibitor & Os01g55770 & NP_001044334 \\
2 & 60S ribosomal protein L18 (RPL18C) & Os03g22180 & NP_001050069 \\
3 & Expressed protein & Os12g37650 & NP_001067038 \\
5 & Polyubiquitin (RUBQ2) & Os02g06640 & NP_001045980 \\
7 & 60S ribosomal protein L24 (RPL24A) & Os07g12250 & NP_001059209 \\
8 & Ania-6a type cyclin (RCY1) & Os01g27940 & NP_001043083 \\
10 & Small nuclear ribonucleoprotein G & Os07g41790 & AAK55776 \\
11 & Dormancy-associated protein & Os11g44810 & NP_001068430 \\
12 & Transferase hexapeptide repeat-containing protein & Os01g18070 & NP_001042763 \\
13 & Expressed protein & Os01g70670 & EEE55952 \\
14 & Dormancy associated protein & Os11g44810 & NP_001068430 \\
\hline
\end{tabular}

${ }^{\mathrm{a}}$ Identical protein.

Table 2 Prediction of molecular characters of OsPUM1-interacting proteins.

\begin{tabular}{lccl}
\hline Interacting-protein & $\mathrm{Mw}(\mathrm{kDa})$ & $\mathrm{pI}$ & Localization $^{\dagger}$ \\
\hline Sigma factor F inhibitor & 9.37 & 15.44 & Chloroplast \\
60S ribosomal protein L18 (RPL18C) & 11.43 & 21.07 & Nucleus/Cytoplasm \\
Expressed protein & 5.71 & 26.81 & Chloroplast \\
Polyubiquitin (RUBQ2) & 7.05 & 51.19 & Cytoplasm \\
60S ribosomal protein L24 (RPL24A) & 10.72 & 18.23 & Cytoplasm \\
Ania-6a type cyclin (RCY1) & 8.93 & 49.13 & Nucleus \\
Small nuclear ribonucleoprotein G & 5.10 & 8.09 & Chloroplast \\
Dormancy-associated protein & 9.45 & 13.57 & Nucleus/Chloroplast \\
Transferase hexapeptide repeat-containing protein & 6.36 & 28.47 & Cytoplasm \\
Expressed protein & 5.56 & 89.31 & Nucleus \\
\hline
\end{tabular}

${ }^{\dagger}$ pSORT prediction.

molecule ${ }^{18}$. However, no characterization of the rice SigF orthologue has been reported. Two ribosomal proteins, RPL18C and RPL24A, were also detected as OsPUM1 partners. In Arabidopsis, the RPL18C interacts with open reading frame VI product (P6) of cauliflower mosaic virus $(\mathrm{CaMV})^{19}$. Interestingly, OsPUM1 also interacts with RUBQ2, a polyubiquitin. Ubiquitin has been reported to be involved in cellular processes such as ribosome biosynthesis, chromatin structure and cell cycle regulation ${ }^{20,21}$. Ubiquitin is also involved in the degradation of phytochrome, plant senescence and stress response ${ }^{22-25}$. RCY1, a cyclin, was also a candidate partner for OsPUM1. In almost all organisms, cyclins play a role in cell cycle regulation, especially during mitotic division. In yeast, overexpression of RCY1 could induce tolerance to $\mathrm{LiCl}$ and $\mathrm{NaCl}^{26}$.

The pSORT software predicted that OsPUM1 is localized in the cytoplasm. In most cases, two interacting proteins are co-localized to perform their action. To confirm the localization of these interacting proteins, the amino acid sequence of each protein was predicted using the identical strategy. As shown in Table 2, the interacting proteins are found in different locations in the plant cell. Three proteins were predicted to be localized in the cytoplasm, RUBQ2, RPL24A, and transferase hexapeptide repeat-containing protein, while RPL18C is a ribosomal protein, so it will also function in the cytoplasm, once the mature ribosomal subunits are translocated there from the nucleus. This in silico study indicates that these four proteins are strong candidates to interact with OsPUM1. However, further analysis such as co-immunoprecipitation (co-IP) or pull down assay is required to confirm this possibility.

In summary, this study underlines the critical function of OSPUM1 that might be involved in a broad extent of biological processes, including not only those related with morphology determination, but is also associated with protein folding and plant immunity. Further study is required to determine the functional role of $O S P U M 1$ and/or interacting genes by using knockout mutants as well as over-expression lines. In addition, subsequent studies for in vitro 
and in planta interaction between OsPUM1 and its partners are justified.

\section{REFERENCES}

1. Gerber AP, Herschlag D, Brown PO (2004) Extensive association of functionally and cytotopically related mRNAs with Puf family RNA-binding proteins in yeast. PLoS Biol 2, 342-74.

2. Hook BA, Goldstrohm AC, Seay DJ, Wickens M (2007) Two yeast PUF proteins negatively regulate a single mRNA. J Biol Chem 282, 15430-8.

3. Forbes A, Lehmann R (1998) Nanos and Pumilio have critical roles in the development and function of Drosophila germline stem cells. Development 125, 679-90.

4. Crittenden SL, Bernstein DS, Bachorik JL, Thompson BE, Gallegos M, Petcherski AG, et al (2002) A conserved RNA-binding protein controls germline stem cells in Caenorhabditis elegans. Nature 417, 660-3.

5. Moore FL, Jaruzelska J, Fox MS, Urano J, Firpo MT, Turek PJ, et al (2003) Human Pumilio-2 is expressed in embryonic stem cells and germ cells and interacts with DAZ (Deleted in AZoospermia) and DAZ-like proteins. Proc Natl Acad Sci USA 100, 538-43.

6. Francischini CW, Quaggio RB (2009) Molecular characterization of Arabidopsis thaliana PUF proteins binding specificity and target candidates. FEBS $J \mathbf{2 7 6}$, 5456-70.

7. Aggarwal P, Yadav RK, Reddy GV (2010) Identification of novel markers for stem-cell niche of Arabidopsis shoot apex. Gene Expr Patterns 10, 259-64.

8. Borges F, Gomes G, Gardner R, Moreno N, McCormick S, Feijó JA, Becker JD (2008) Comparative transcriptomics of Arabidopsis sperm cells. Plant Physiol 148, 1168-81.

9. Abbasi N, Park Y-I, Choi S-B (2011) Pumilio Puf domain RNA-binding proteins in Arabidopsis. Plant Signaling Behav 6, 364-8.

10. Fields S, Song OK (1989) A novel genetic system to detect protein-protein interactions. Nature 340, 245-6.

11. Kikuchi S, Satoh K, Nagata T, Kawagashira N, Doi K, Kishimoto N, Yazaki J, Ishikawa M, et al (2003) Collection, mapping, and annotation of over 28,000 cDNA clones from japonica rice. Science 301, 376-9.

12. Wheelan SJ, Church DM, Ostell JM (2001) Spidey: a tool for mRNA-to-genomic alignments. Genome Res 11, 1952-7.

13. Bjellqvist B, Hughes GJ, Pasquali C, Paquet N, Ravier F, Sanchez J-C, et al (1993) The focusing positions of polypeptides in immobilized $\mathrm{pH}$ gradients can be predicted from their amino acid sequences. Electrophoresis 14, 1023-31.

14. Sigrist CJA, Cerutti L, de Castro E, LangendijkGenevaux PS, Bulliard V, Bairoch A, Hulo N (2010) PROSITE, a protein domain database for functional characterization and annotation. Nucleic Acids Res 38, 161-6.

15. Gietz D, St Jean A, Woods RA, Schiestl RH (1992) Improved method for high effieciency transformation of intact yeast cells. Nucleic Acids Res 20, 1425.

16. Breeden L, Nasmyth K (1995) Regulation of the yeast HO gene. Cold Spring Harbor Symp Quant Biol 50, 643-50.

17. Ling M, Merante F, Robinson BH (1995) A rapid and reliable DNA preparation method for screening a large number of yeast clones by polymerase chain reaction. Nucleic Acids Res 23, 4924-5.

18. Chen P, Ruiz RE, Li Q, Silver RF, Bishai WR (2000) Construction and characterization of a Mycobacterium tuberculosis mutant lacking the alternate sigma factor gene, sigF. Infect Immun 68, 5575-80.

19. Leh V, Yot P, Keller M (2000) The cauliflower mosaic virus translational transactivator interacts with the $60 \mathrm{~S}$ ribosomal subunit protein L18 of Arabidopsis thaliana. Virology 266, 1-7.

20. Finley D, Bartel B, Varshavsky A (1989) The tail of ubiquitin precursors are ribosomal proteins whose fusion to ubiquitin facilitates ribosome biogenesis. $\mathrm{Na}$ ture 338, 394-401.

21. Rechsteiner M (1991) Natural substrates of the ubiquitin proteolytic pathway. Cell 66, 615-8.

22. Jabben M, Shanklin J, Vierstra RD (1989) Ubiquitinphytochrome conjugates. Pool dynamics during in vivo phytochrome degradation. J Biol Chem 264, 4998-5005.

23. Becker F, Buschfeld E, Schell J, Bachmair A (1993) Alter response to viral infection by tobacco plants perturbed in ubiquitin system. Plant J 3, 875-81.

24. Belknap WR, Garbarino J (1996) The role of ubiquitin in plant senescence and stress response. Trends Plant Sci 1, 331-5.

25. Durzan DJ (1996) Protein ubiquitination in diploid parthenogenesis and early embryos of Norway spruce. Int J Plant Sci 157, 17-26.

26. Forment J, Naranjo MA, Roldán M, Serrano R, Vicente O (2002) Expression of Arabidopsis SR-like splicing proteins confers salt tolerance to yeast and transgenic plants. Plant J 30, 511-9. 